\title{
In Silico Molecular Modeling and Docking Studies on the Leishmanial Tryparedoxin Peroxidase
}

\author{
Ozal Mutlu* \\ Marmara University, Faculty of Arts and Sciences; Department of Biology; Kadikoy-Istanbul-Turkey
}

\begin{abstract}
Leishmaniasis is one of the most common form of neglected parasitic disease that affects about 350 million people worldwide. Leishmanias have a trypanothione mediated hydroperoxide metabolism to eliminate endogenous or exogenous oxidative agents. Both of 2-Cys peroxiredoxin (Prx) and glutathione peroxidase type tryparedoxin peroxidase $(P x)$ are the terminal enzymes in the trypanothione dependent detoxification system. Therefore absence of trypanothione redox system in mammals and the sensitivity of trypanosomatids against oxidative stress, enzymes of this pathway are drug targets candidates. In this study, 3D structure of tryparedoxin peroxidase (2-Cys peroxiredoxin type) from Leishmania donovani (LdTXNPx) was described by homology modeling method based on the template of tryparedoxin peroxidase from Crithidia fasciculata and selected compounds were docked to the active site pocket. The quality of the 3D structure of the model was confirmed by various web based validation programs. When compared secondary and tertiary structure of the model, it showed a typical thioredoxin fold containing a central beta-sheet and three alpha-helices. Docking study showed that the selected compound 2 (CID 16073813 ) interacted with the active site amino acids and binding energy was $-118.675 \mathrm{kcal} / \mathrm{mol}$.
\end{abstract}

Key words: Homology modeling, docking, Leishmania donovani, tryparedoxin peroxidase, hydroperoxide metabolism

\section{INTRODUCTION}

Leishmaniasis is caused by trypanosomatid protozoan parasites that belong to the genus Leishmania and it is one of the most neglected parasitic diseases that affect about 350 million people together with two million new cases yearly. Leishmaniasis is seen frequently in Southeast Asia, Africa, South America, including mostly Brazil and Mediterranean countries (WHO 2010). Leishmaniasis has three clinical forms seen in humans, which are cutanous leishmaniasis $(L$. major, $L$. tropica and $L$. mexicana); visceral leishmaniasis ( $L$. donovani and $L$. infantum) that is responsible from thousands of deaths each year and mucocutaneous leishmaniasis (L. braziliensis).
The disease is transmitted by infected female sandflies to the vertebrate hosts where the parasites infect and reproduce within their macrophages (Desjeux 2001, Pavli et al. 2010).

Parasites are exposed to various reactive oxygen species (superoxide anion radical, hydrogen peroxide, peroxynitrite and the hydroxyl radical) generated by the host defense system, or endogenously by parasite itself (Krauth-Siegel and Combos 1999, Müller et al. 2003, Krauth-Siegel and Comini 2008). However, they have adapted to survive and replicate inside the host macrophages, which produce ROS and NO derivatives for the destruction of microorganism normally (Assche et al. 2011). This is because of all the trypanosomatids have a trypanothione mediated

*Author for correspondence: ozal.mutlu@marmara.edu.tr 
hydroperoxide metabolism to eliminate endogenous, or exogenous oxidative agents. Detoxification of the hydroperoxidases comprises an electron transfer cascade that contains trypanothione, trypanothione reductase, tryparedoxin and tryparedoxin peroxidases (2-Cys peroxiredoxin and glutathione peroxidase type tryparedoxin peroxidases) enzymes (Müller et al. 2003). All the trypanosomatid protozoan parasites bear this thiol redox pathway and share same biochemical, morphological and molecular characteristics (Krauth-Siegel and Comini 2008). Due to lack of glutathione reductase, catalse, thioredoxin reductase and glutathione peroxidase mechanisms in the parasites, which are found in their mammalian hosts, trypanothione is the only system that protects parasites from the oxidant damage and also toxic effects of the heavy metals (Flohe et al. 1999, Krauth-Siegel and Comini 2008).

Both of 2-Cys peroxiredoxin (Prx) and glutathione peroxidase type tryparedoxin peroxidase $(\mathrm{Px})$ are the terminal enzymes in the trypanothione dependent detoxification system in trypanosomatids. Electron transfer occurs between the trypanothione reductase and tryparedoxin mediated by trypanothione. Tryparedoxin peroxidase accepts an electron from the tryparedoxin for the reduction of the hydrogen peroxide and peroxynitrite (Irigoin et al. 2008). However, some other compounds could be as substrates for the 2-Cys peroxiredoxin (Prx) such as linoleic acid hydroperoxide (Nogoceke et al. 1997). Studies have indicated that the tryparedoxin peroxidases involved in the trypanothione dependent efflux system are essential, for the parasite survival and they have similar activities and cellular localizations (Dumas et al. 1997, Tovar et al. 1998, Wilkinson et al. 2000, Tetaud et al. 2001, Castro et al. 2002). RNA interference studies have shown that the inhibition of the enzymes cause impaired cell growth, enhanced sensitivity towards $\mathrm{H}_{2} \mathrm{O}_{2}$ and other oxidants and cell death (Wilkinson et al. 2003, Lin et al. 2005, Schlecker et al. 2005). However overexpression reveals enhanced resistance towards arsenite, hydroperoxide and NO (Lin et al. 2005, Castro et al. 2002, Pineyro et al. 2008). In addition, further proteomic analysis also reveals the expression of the eleveted levels of the trypanothione redox pathway proteins in both of trypanosomatid parasites Trypanosoma sp. and Leishmania sp. in different life stages (Daneshavar et al. 2012, Diaz et al. 2011). Some other findings also show that there is a positive correlation between the elevated levels of tryparedoxin peroxidase with the enhanced antioxidant defenses and resistance to conventional drugs (Wyllie et al. 2010).

Thus, existing treatments for the leishmaniasis are limited because they are based on a few drugs that have some side effects and increasing resistance to conventional drugs. This necessitates a continuous demand for novel drugs. In drug research process, selecting potential drug targets should play an essential role in the parasite survival and also should have a significant structural and functional difference from its mammalian counterparts (Sing et al. 2012). Together with the absence of trypanothione redox system in the mammals and the sensitivity of trypanosomatids against oxidative stress, enzymes of the pathway are attractive candidates for drug targets (KrauthSiegel et al. 2005, Jaeger and Flohe 2006, Pineyro et al. 2008).

For computational drug design studies, 3D structure of the interest protein should be known. Crystal structure of the tryparedoxin peroxidase from Trypanosoma cruzi (TcTXNPx) and $C$. fasciculata were dissolved by the method of X-ray diffraction (Alphey et al. 2000, Pineyro et al. 2005). However, currently there is no solved crystal structure for tryparedoxin peroxidase from the genus of Leishmania. Computational homology, based modeling methods are the best and fast techniques to predict the protein structure from a known 3D template when there is no solved structure (Hillisch et al. 2004, Werner et al. 2012). Templates for homology modeling should be selected from the evolutionary closest protein family and have a sequence identity higher than $50 \%$, which is ideal for drug design (Hillisch et al. 2004, Bishop et al. 2008). Computer-aided drug discovery process comprises three main steps; prediction of the $3 \mathrm{D}$ structure of the protein, prediction of the possible ligands and docking procedures and biological testing of the drug candidates (Huang et al. 2010).

In this work, 3D structure of tryparedoxin peroxidase (2-Cys peroxiredoxin type) from $L$. donovani (LdTXNPx) was described by homology modeling method based on the template of tryparedoxin peroxidase from C. fasciculata and docking studies was performed with selected five compounds. Homolog modeling study included template identification, model building, followed by the side chain refinement and validation of the resultant protein structure. 


\section{MATERIALS AND METHODS}

Target sequence, template identification and sequence alignment

The amino acid sequence of the target tryparedoxin peroxidase for $L$. donovani was obtained from the (National Center for Biotechnology Information, NCBI) database with the accession number of AAK00633. For template identification, NCBI BLAST tool was used and two types of BLAST (Basic Local Alignment search Tool) protein-protein BLAST and PSIBLAST (Position Specific Iterated-BLAST) was performed for template selection by searching against database Protein Data Bank proteins. At the end of comparative searching, one of the homolog structure that had the best score was selected as a template protein. Template protein PDB file and amino acid sequence in FASTA format was downloaded from the Protein Data Bank. Multiple sequence alignment of the template and target proteins were analyzed by ClustalW2 (Larkin et al. 2007) from the EBI (European Bioinformatics Institute) sequence analysis tools.

\section{Homology model building and refinement}

A homology model of the $L d$ TXNPx enzyme was constructed by using the software of Modeller v9.11 (Eswar et al. 2006). Model was built according to the target sequence, an alignment file and 3D structure of the template protein that was obtained from the Protein Data Bank (PDB). Refinement of the model was done by performing energy minimization by HyperChem software (Hypercube Inc. Gainesville, FL, USA). Secondary structure of the protein was estimated by web based program SOPMA (Geourjon and Deleage 1995) and NCBI CDD were used to determine the domains. The PDB file of the resultant model has been deposited in the Protein Model Data Base (PMDB) with ID number of PM0078249. All generated structures were visiulazed by MolSoft ICM-Browser (MolSoft LLC, La Jolla, CA, USA).

\section{Model validation and bioinformatics}

Stereochemistry of the model was checked by and RAMPAGE (Lovell et al. 2007). Structure quality of the protein was analyzed by ProSA (Wiederstein and Sippl 2007) and Protein Structure Validation Suite, PSVS 1.3. Statics of non-bonding interactions was performed by ERRAT (Colovos and Yeates 1993). Various physical and chemical parameters for the modeled protein included the molecular weight, theoretical pI, amino acid composition, extinction coefficient, aliphatic index and grand average of hydropathicity (GRAVY), which were computed by ProtParam tool from ExPASy.

\section{Docking studies}

In docking study, compounds were searched for the leishmanial activity (IC50, etc $\leq 1 \mathrm{nM}$ ) by using PubChem BioAssay database from NCBI. Three dimensional structures of the selected compounds were retrieved as SD file format. They were 3-naphthalen-2-yloxy-1,2-benzothiazole 1,1dioxide (Compound ID: 790223), 3-methoxy-5-(3methylsulfanyl-1,2,4-triazin-5-yl)-1,2,4-triazine (Compound ID: 2865728), 6,6-dimethyl-1[3-[(3-propan-2-ylphenoxy)methyl]phenyl]-1,3,5triazine-2,4-diamine (Compound ID: 16073813), 4-methyl-7-[(1-oxido-1-oxo-1,2-benzothiazol-3yl)sulfanyl]chromen-2-one (Compound ID: 46912206) and N-(4,6-dimethylpyrimidin-2-yl)4,5,7-trimethylquinazolin-2-amine (Compound ID: 53325662). Docking study was performed by using Molegro Virtual Docker software (Thomsen and Christensen 2006) and docking results were analyzed by Molegro Molecular Viewer 2.5.0 (Molegro ApS, Aarhus, Denmark).

\section{RESULTS AND DISCUSSION}

Template identification and sequence alignment BLAST analysis was performed of tryparedoxin peroxidase from $L$. donovani ( $L d \mathrm{TXNPx}$ ) against Protein Data Bank proteins (PDB). The three top results belonged to tryparedoxin peroxidase from Crithidia fasciculata (PDB ID: 1E2Y, Identities = 134/184 (73\%), E-value 4e-101), tryparedoxin peroxidase (Txnpx) from Trypanosoma cruzi (PDB ID: 1UUL, Identities: 137/196 (70\%), Evalue: 3e-101) and peroxiredoxin I from Schistosoma mansoni (PDB ID: 3ZVJ, Identities = 117/181 (65\%), E-value: 8e-86). Homology models having sequence identities between 30 and $50 \%$ could be used in structure-based target assessment and site-directed mutagenesis. Sequence identity above $50 \%$ was ideal for structure-based drug design and target assessment, site directed mutagenesis and assignment of protein function (Hillisch et al. 2004). The sequence identity between the selected template and target was $73 \%$, hence models could be used directly in drug design studies. Distance tree of the BLAST results showed that these proteins were closely related. According to the BLAST search, 
the closest enzyme was from $C$. fasciculata (PDB ID: 1E2Y, Alphey et al. 2000), which was selected as a template for homology modeling. Figure 1 shows multiple sequence alignments of the tryparedoxin peroxidases from $L$. donovani, C. fasciculata and T. cruzi. 1 25

C. fasciculata
L. donovani
T. cruzi
MSCGNAKINCRAPPFEEVALMPNGSFKKISLAAYKGKWVVLFFYPLDETE MSCGEAEDLHPAPDFNETALMPNGT EKKVALTSYKGKWLVLFFYPMDETE

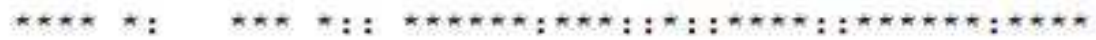

75

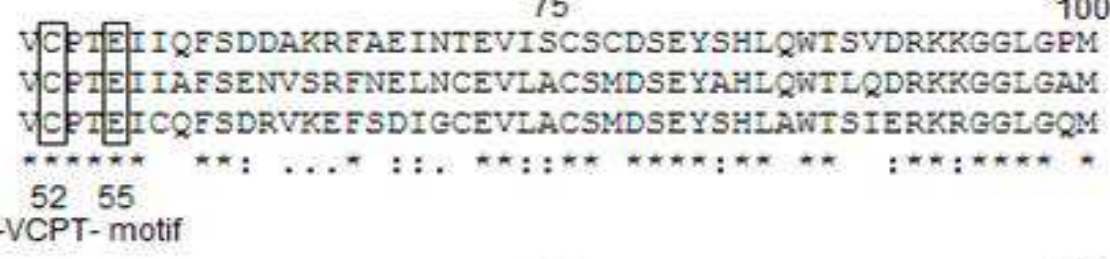

\section{C. fasciculata \\ L. donovani \\ T. cruzi}

\section{0}

125

C. fasciculata

L. donovani

T. cruzi

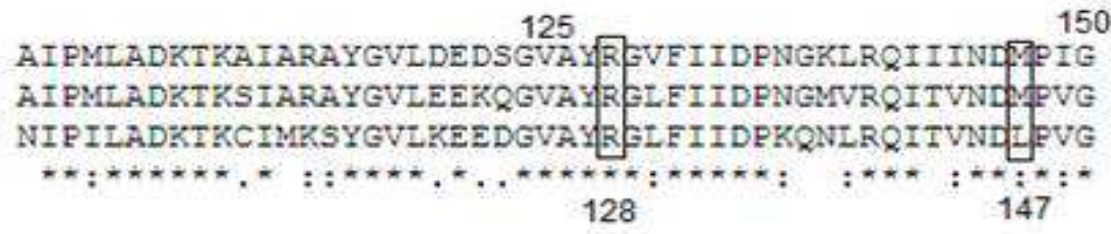

175

C. fasciculata
L. donovani
T. cruzi

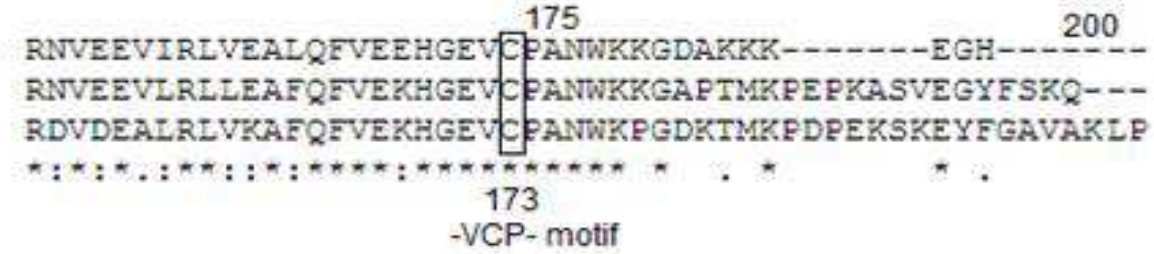

Figure 1 - Multiple sequence alignment of the tryparedoxin peroxidases from Crithidia fasciculata, Leishmania donovani and Trypanosoma cruzi. Boxes show active site residues. (*) conserverd residues.

\section{Structural features}

All the peroxiredoxins shared strictly conserved cysteines at the position of 52 and 173 at the Nand $\mathrm{C}$ - terminal regions. They are often found in a -VCPT- and -VCP- motif (Alphey et al. 2000). These cysteine residues are involved in catalytic activity thus essential for activity (Flohe et al. 2002). As shown in the alignment, all four enzymes had these conserved regions. Because of having two Cys residues at the $\mathrm{N}$ - and $\mathrm{C}$ - terminal motif, they are called as 2-Cys peroxiredoxins. Secondary structure predictions showed that the enzyme had 61 residues in $\alpha$-helix (30.65\%), 43 residues in extended strands $(21.61 \%), 18$ in $\beta$-turn $(9.05 \%)$ and others $(77,38.69 \%)$ in random coil form. Overall structure comprised four $\alpha$-helices, seven $\beta$-strands and two $310^{-}$helix $(\theta 1$ and $\theta 2$ ). $N$ terminus of the structure began with a $\beta$-hairpin ( $\beta 1$ and $\beta 2$ ), followed by a section of $3_{10}$-helix $(\theta 1)$, two $\beta \alpha \beta$ units ( $\beta 3-\alpha 1-\beta 4-\alpha 2-\beta 5), 3_{10}$-helix ( $\theta 2$ ) found between $(\alpha 2-\beta 5)$, then $\alpha 3$, a $\beta$-hairpin $(\beta 6$ and $\beta 7)$ and $\alpha 4$ before $\mathrm{C}$ - terminal. This secondary structure highly resembled to the secondary structure of the template enzyme, which had same folding features (Alphey et al. 2000). Like in C. fasciciulata TXNPx, Cys52 was found before $\alpha 1$ and Cys173 after that $\alpha 4$ helices.

The 3D structure of the model indicated that the enzyme comprised a typical thioredoxin fold that contained a central $\beta$-sheet $(\beta 7, \beta 6, \beta 3, \beta 4)$ and three $\alpha$ helices ( $\alpha 1, \alpha 4$ and $\alpha 3$ ) (Alphey et al. 2000) (Fig. 2). Hence, it has been classified as a member of the thioredoxin superfamily and glutathione peroxidase-like family according to the domain search and structural results. When compared 3D structures of $C$. fasciculata TXNPx and $L$. donovani TXNPx, they fit with a high similarity. Superposition of the $\mathrm{C}_{\alpha}$ traces of the $C f$ TXNPx and $L d$ TXNPx had RMSD of $0.4223 \AA$ and 179 atoms overlapping (Fig. 3). 


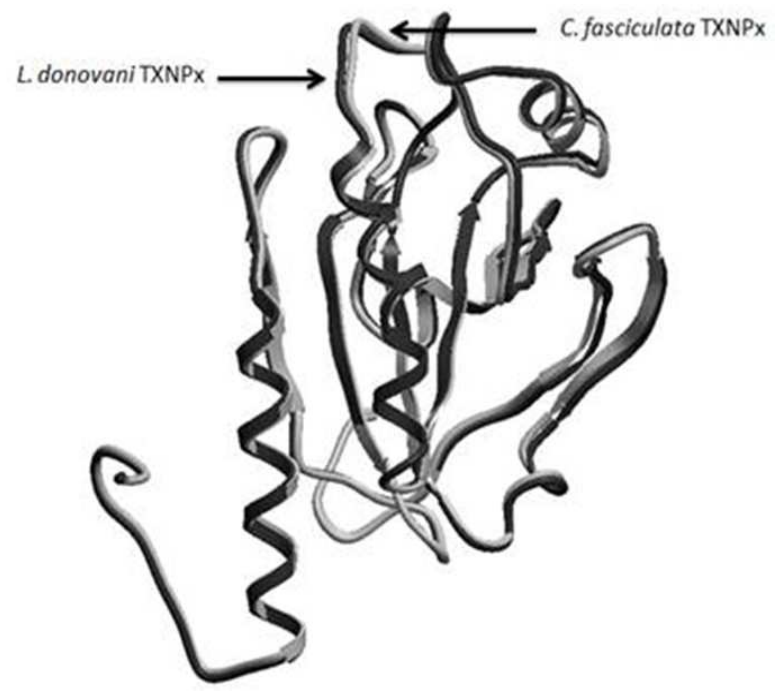

Figure 2 - Superposition of the template (Crithidia fasciculata tryparedoxin peroxidase, PDB ID 1E2Y) and the target (Leishmania donovani tryparedoxin peroxidase) proteins according to the $\mathrm{C}_{\alpha}$ traces (RMSD $=0.4223 \AA$ ). Black color represents $L$. donovani TXNPx and white one is for $C$. fasciculata TXNPX.

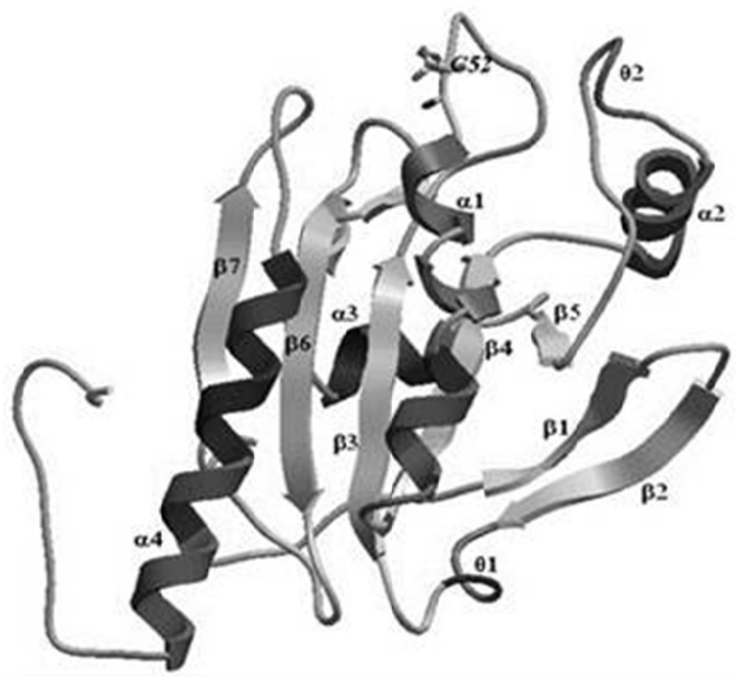

Figure 3 - 3D structural model of Leishmania donovani tryparedoxin peroxidase. Helix forms represent $\alpha$-helices and large arrows for $\beta$-hairpin structures. $N$ - terminus of the structure begins with a $\beta$-hairpin ( $\beta 1$ and $\beta 2$ ) and terminates with $\alpha 4$ before $C$ - terminal. Structure shows a typical thioredoxin fold contains a central $\beta$-sheet $(\beta 7, \beta 6, \beta 3, \beta 4)$ and three $\alpha$ helices $(\alpha 1, \alpha 4$ and $\alpha 3)$ around the sheet.
The peroxidase active site comprises Cys52 and Cys173, which are directly responsible for peroxidase activity (Hirotsu et al. 1999, Alphey et al. 2000, Schröder et al. 2000). In C. fasciciulata TXNPx, Cys52 accepts a hydrogen bond from $\operatorname{Arg} 128$, donates to the carbonyl of Met147 and carboxylate of Glu55. Arg128 is thought to be stabilizing the ionized state of Cys52, lower its $\mathrm{p} K_{\mathrm{a}}$ and increases the activity. Same active site residues are also found in $L$. donovani TXNPx at the same positions. $L d \mathrm{TXNPx}$ comprises three different pockets. Two of them are very important because of having active site residues of Cys52 and Cys173. The first pocket is located between $\beta 3-\alpha 3$ and $\alpha 1$ and interacted with some of the active site residues (Cys52, Met147 and Arg128) and also with others such as Tyr44, Leu46, Thr49, Ile56, Ala58, Pro148, Gly150 and Glu154 residues. The second one is found at the Cterminal and interacts with Arg158, Leu159, Ala162, Phe163, Val166, Cys173 and Trp177 residues.

\section{Structure validation}

Constructed new model, which was called as $L d T X N P x$, was checked for stereochemistry, energy profiles, potential errors, non-bonding interactions and some physical and chemical properties. According to the ProtParam results, protein model is about $22.2 \mathrm{kDa}$, pI is 7.55 , have 22 negatively and 23 positively charged residues, extinction coefficient was 25815 , aliphatic index was 79.40 and grand average of hydropathicity was -0.108 . According to the RAMPAGE (Lovell et al 2007), results $89.6 \%$ of residues (155) were in favored, $7.5 \%$ (13) in allowed and $2.9 \%$ (5) in outlier region in ramachandran plot that analyzed peptide dihedral angles. ERRAT was used to predict non-bonding interactions by examining the statistics of pair wise atomic interactions $(\mathrm{CC}, \mathrm{CN}$, $\mathrm{CO}, \mathrm{NN}, \mathrm{NO}$, and OO). Program gave a result of 93.865 as overall quality factor, which meant only 10 residues showed incorrect determined regions (Colovos and Yeates 1993). ProSA calculated overall protein quality based on the scores of all experimentally determined protein chains available in PDB. The program gives $\mathrm{z}$-score and a plot of the residue energies (Wiederstein and Sippl 2007). Z-score of $L d T X N P x$ was -6.46. It was within the range of scores typically found for the proteins of similar size and also close to the zscore value of the CfTXNPx that was -7.4. The energy plot calculated the average energy over 
each 40-residues fragment and overall negative values of the model indicated the structure is nonproblematic. Protein Structure Validation Suite (PSVS 1.3) was also used to analyze overall model quality based on the global quality scores of four different structure validation softwares, which were Verify3D, ProsaII, Procheck and MolProbity (Table1). All quality check results showed that the model was close to the native form and the environment profile of the structure was good.

Table 1 - Protein structure validation suite (PSVS) results for the global quality of the protein model

Program Verify ProsaII Procheck ${ }^{* *}$ Procheck MolProbity

\begin{tabular}{|c|c|c|c|c|c|}
\hline & 3D & & & $(\text { all })^{* * *}$ & Clashscore \\
\hline $\begin{array}{l}\text { Raw } \\
\text { score }\end{array}$ & 0.43 & 0.50 & -0.66 & -0.68 & 8.31 \\
\hline Z-score* & 0.48 & -0.62 & -228 & -4.02 & 0.10 \\
\hline
\end{tabular}

${ }^{*}$ With respect to mean and standard deviation for a set of $252 \mathrm{X}$ ray structures $<500$ residues, of resolution $<=1.80 \AA$, R-factor $<=0.25$ and $\mathrm{R}$-free $<=0.28$; a positive value indicates a 'better' score. ${ }^{* *}$ Selected residues: $1 \mathrm{~A}-178 \mathrm{~A}$

\section{Docking studies}

Molegro Virtual Docker uses a molecular docking algorithm called as MolDock which has higher docking accuracy than well known programs (GOLD, Surflex, FlexX and Glide). The scoring function, Escore, is defined by the summation of Einter (ligand-protein interaction energy) and Eintra (internal energy of the ligand) (Thomsen and Christensen 2006). Thus docking method use an energy-based scoring function, lower energy scores represent better protein-ligand bindings compared to higher energy values (Thomsen and Christensen 2006). Previous studies showed that the compound 2 (CID 16073813) inhibited the dihydrofolate reductase (DHFR) from $L$. major $\left(\mathrm{K}_{\mathrm{i}}\right.$ : 0.17378) (Booth, et al 1987), compound 1 (CID 53325662) had antiprotozoal activity against $L$. donovani (LC50: $32 \mu \mathrm{M})$ and antitrypanosomal activity against $T$. cruzi (LC50: $8 \mu \mathrm{M})$ by inhibiting trypanothione reductase enzyme (Holloway et al. 2009), compound 3 (CID 46912206) and 4 (CID 790223) had an inhibition on L. mexicana Pyruvate Kinase and Compound 5
(CID 2865728) had antitrypanosomal activity against bloodstream form of T. brucei (EC50: 5.7 $\mu \mathrm{M})$ (Nhu et al. 2010), antimalarial activity against erythrocytic stage of Plasmodium falciparum 3D7 (EC50: $0.18 \mu \mathrm{M}$ ) (Nhu et al. 2010) and antiprotozoal activity against $L$. donovani MHOM/ET/67/L82 amastigotes (IC50: $8 \mu \mathrm{M}$ ) (Holloway et al. 2009). According to docking results, all biological active compounds had interaction with the active site residues (Cys52, Arg128 and Glu55) (Hirotsu et al. 1999, Alphey et al. 2000, Schröder et al. 2000). Except compound 5, others had nearly same MolDock scores. Compound 2 had the highest MolDock score which was $-118.675 \mathrm{kcal} / \mathrm{mol}$. However there was no $\mathrm{H}$ bond interaction with catalytic residues. Compound 5 had the highest $\mathrm{H}$ bond score $(-11.128 \mathrm{kcal} / \mathrm{mol})$ and $\mathrm{H}$ bond interaction with important catalytic residue Arg128. Compound 3 and 5 had $\mathrm{H}$ bond interaction with Arg128 too (Table 2).

\section{CONCLUSIONS}

In Leishmania species, reductions of hyperoxidases are provided directly by the tryparedoxin peroxidase activities. Because of parasites are sensitive against to the oxidative stress, enzymes involved in this pathway are attractive targets for drugs. In the absence of experimental structure, in silico homology modeling of the proteins provides a fast and costeffective method in the structural based drug discovery process. In this study, a homology model of tryparedoxin peroxidase from $L$. donovani ( $L d \mathrm{TXNPx}$ ) was determined based on the template by the closely related trypanasomatid, C. fasciculata. When compared with the secondary and tertiary structure of the model, it showed a typical thioredoxin fold containing a central $\beta$-sheet $(\beta 7, \beta 6, \beta 3, \beta 4)$ and three $\alpha$ helices $(\alpha 1, \alpha 4$ and $\alpha 3)$. Docking study was indicated that the compound 2 had the highest docking score and could provide a scaffold for further drug design works. 
Table 2 - Docking results of the selected compounds.

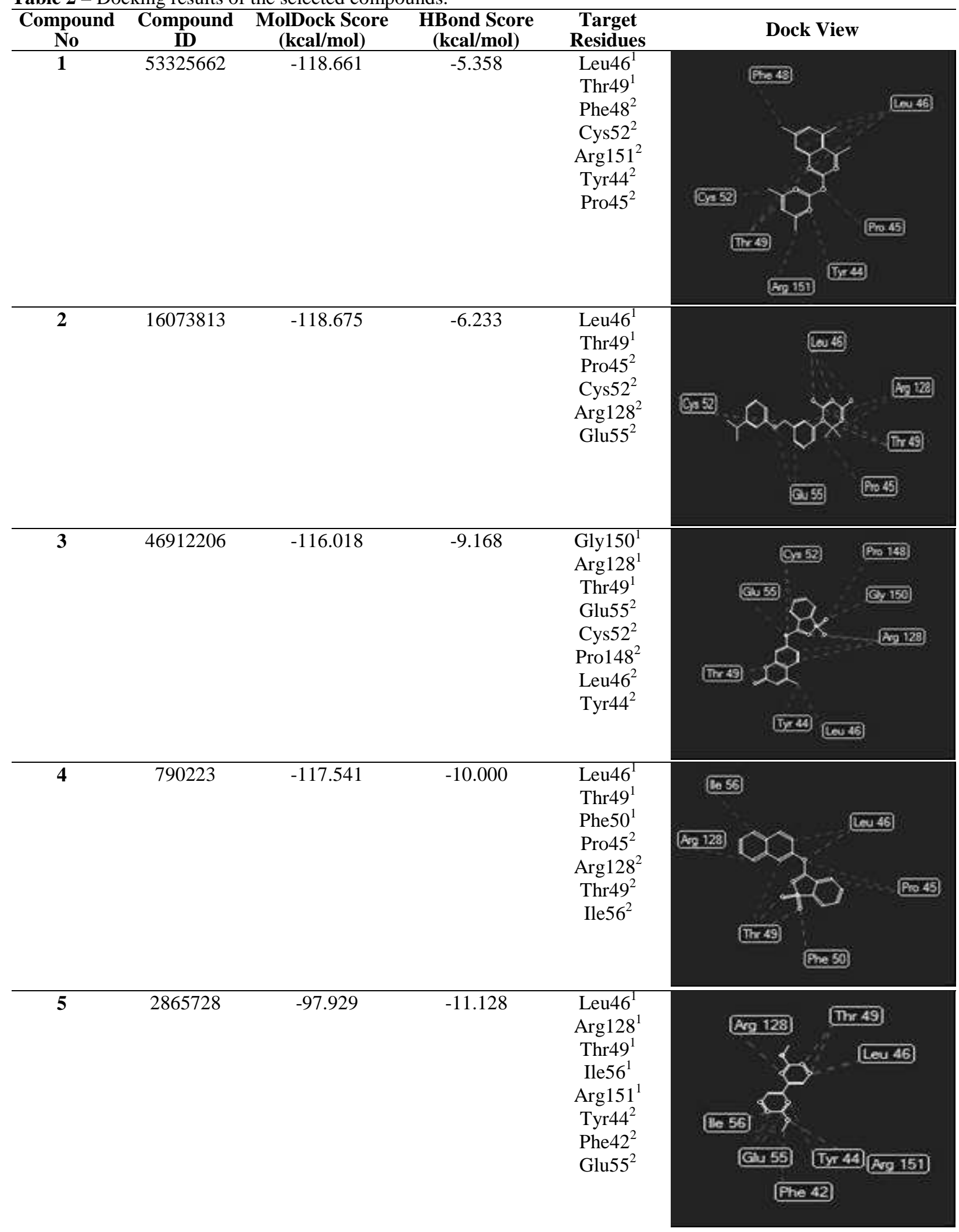




\section{REFERENCES}

Alphey MS, Bond CS, Tetaud E, Fairlam AH, Hunter WN. The structure of reduced tryparedoxin peroxidase reveals a decamer and insight into reactivity of 2Cys-peroxiredoxins. J Mol Biol. 2000; 300: 903-916.

Assche TM, Deschacht M, Luz RA, Maes L, Cos P. Leishmania-macrophage interactions: Insights into the redox biology. Free Radi Biol Med. 2011; 51: 337-351.

Bishop OT, de Beer TAP, Joubert F. Protein homology modeling and its use in South Africa. S Afr J Sci. 2008; 104: 2-6.

Booth RG, Selassie CD, Hansch C, Santi DV. Quantitative structure-activity relationship of triazineantifolate inhibition of Leishmania dihydrofolate reductaseand cell growth. J Med Chem.1987; 30(7): 1218-24.

Castro H, Sousa C, Santos M, Cordeiro-da-Silva A, Flohé L, Tomas AM. Complementary antioxidant defense by cytoplasmic and mitochondrial peroxiredoxins in Leishmania infantum. Free Radi Biol Med. 2002; 33: 1552-1562.

Colovos C, Yeates TO. Verification of protein structures: patterns of nonbonded atomic interactions. Protein Science. 1993; 2: 1511-1519.

Cuervo P, de Jesus JB, Junqueira M, Mendonca-Lima L, Gonzalez LJ, Betancourt L, Grimaldi G, Domonte GB, Fernandes O, Cupolillo E. Proteome analysis of Leishmania (Viannia) braziliensis by twodimensional gel electrophoresis and mass spectrometry. Mol Biochem Parasitol. 2007; 154: 621.

Daneshvar H, Wyllie S, Phillips S, Hagan P, Burchmore R. Comparative proteomics profiling of a gentamicin-attenuated Leishmania infantum cell line identifies key changes in parasite thiol-redox metabolism. J Proteom. 2012; 75: 1463-1471.

Desjeux P. The increase in risk factors for leishmaniasis worldwide. Trans R Soc Trop Med Hyg. 2001; 95: 239-243.

Díaz ML, Solari A, González CI. Differential expression of Trypanosoma cruzi I associated with clinical forms of Chagas disease: overexpression of oxidative stress proteins in acute patient isolate. $J$ Proteom. 2011; 74: 1673-1682.

Dumas C, Ouellette M, Tovar J, Cunningham ML, Fairlamb AH, Tamar S, Olivier M, Papadopoulou B. Disruption of the trypanothione reductase gene of Leishmania decreases its ability to survive oxidative stress in macrophages. EMBO J. 1997; 16: 25902598.
Eswar N, Marti-Renom MA, Webb B, Madhusudhan MS, Eramian D, Shen M, Pieper U, Sali A. Comparative Protein Structure Modeling With MODELLER. Current Protocols in Bioinformatics. John Wiley \& Sons, Inc; 2006. Supplement 15.

Flohe L, Hecht H, Steinert P. Glutathione and trypanothione in parasitic hydroperoxide metabolism. Free Radic Biol Med. 1999; 27: 966984.

Flohé L, Budde H, Bruns K, Castro H, Clos J, Hofmann B, Kansal-Kalavar S, Krumme D, Menge U, PlankSchumacher K, Sztajer H, Wissing J, Wylegalla C, Hecht HJ. Tryparedoxin peroxidase of Leishmania donovani: molecular cloning, heterologous expression, specificity, and catalytic mechanism. Arch Biochem Biophys. 2002; 397: 324-335.

Geourjon C, Deleage G. SOPMA: significant improvements in protein secondary structure prediction by consensus prediction from multiple alignments. Comput Appl Biosci. 1995; 11(6): 681684.

Hillebrand H, Schmidt A, Krauth-Siegel RL. A second class of peroxidases linked to the trypanothione metabolism. J Biol Chem. 2003; 278: 6809-6815.

Hillisch A, Pineda LP, Hilgenfeld R. Utility of homology models in the drug discovery process. Drug Discov Today. 2004; 9(15): 659-69.

Hirotsu S, Abe Y, Okada K, Nagahara N, Hori H, Nishino T, Hakoshima T. Crystal structure of a multifunctional 2-Cys peroxiredoxin heme-binding protein $23 \mathrm{kDa}$ /proliferation-associated gene product. PNAS. 1999; 96(22): 12333-12338.

Holloway GA, Charman WN, Fairlamb AH, Brun R, Kaiser M, Kostewicz E, Novello PM, Parisot JP, Richardson J, Street IP, Watson KG, Baell JB. Trypanothione reductase high-throughput screening campaign identifies novel classes of inhibitors with antiparasitic activity. Antimicrob Agents Chemother. 2009; 2824-2833.

Huang H, Yu HW, Chen C, Hsu C, Chen H, Lee K, Tsai F, Chen CF. Current developments of computeraided drug design. J Taiwan Inst Chem Eng. 2010; 41: 623-635.

Irigoín F, Cibils L, Comini MA, Wilkinson SR, Flohé L, Radi R. Insights into the redox biology of Trypanosoma cruzi: Trypanothione metabolism and oxidant detoxification. Free Radi Biol Med 2008; 45: 733-742.

Jaeger T, Flohé L. The thiol-based redox networks of pathogens: unexploited targets in the search for new drugs. Biofactors. 2006; 27: 109-120. 
Krauth-Siegel RL, Coombs GH. Enzymes of parasite thiol metabolism as drug targets. Parasitol Today. 1999; 15(10): 404-409.

Krauth-Siegel RL, Bauer H, Schirmer RH. Dithiol proteins as guardians of the intracellular redox milieu in parasites: old and new drug targets in trypanosomes and malaria-causing plasmodia. Chem Int. 2005; 44: 690-715.

Krauth-Siegel RL, Comini MA. Redox control in trypanosomatids, parasitic protozoa with trypanothione-based thiol metabolism. Biochim Biophys Acta. 2008; 1780:1236-1248.

Larkin MA, Blackshields G, Brown NP, Chenna R, McGettigan PA, McWilliam H, Valentin F, Wallace IM, Wilm A, Lopez R, Thompson JD, Gibson TJ, Higgins DG. Clustal W and Clustal X version 2.0. Bioinform. 2007; 23: 2947-2948.

Lin YC, Hsu JY, Chiang SC, Lee ST. Distinct overexpression of cytosolic and mitochondrial tryparedoxin peroxidases results in preferential detoxification of different oxidants in arseniteresistant Leishmania amazonensis with and without DNA amplification. Mol Biochem Parasitol. 2005: 142: 66-75.

Lovell SC, Davis IW, Arendall WB, de Bakker PIW, Word JM, Prisant MG, Richardson JS, Richardson DC. Structure validation by Calpha geometry: phi, psi and $\mathrm{C}$ beta deviation. Proteins:Struct Funct Genet. 2002; 50: 437-450.

Muller S, Liebau E, Walter RD, Krauth-Siegel RL. Thiol-based redox metabolism of protozoan parasites. Trends Parasitol. 2003; 19(7): 320-328.

Nhu D, Duffy S, Avery VM, Hughes A, Baell JB. Antimalarial 3-arylamino-6-benzylamino-1,2,4,5tetrazines. Bioorg Med Chem Lett. 2010; 20: 44964498.

Nogoceke E, Gommel DU, Kiess M, Kalisz HM, Flohé L. A unique cascade of oxidoreductases catalyses trypanothione-mediated peroxide metabolism Crithidia fasciculata. Biol Chem. 1997; 378: 827836.

Pavli A, Maltezou HC. Leishmaniasis, an emerging infection in travelers. Int J Infec Dis. 2010; 14: 10321039.

Piñeyro MD, Pizarro JC, Lema F, Pritsch O, Cayota A, Bentley GA, Robello C. Crystal structure of the tryparedoxin peroxidase from the human parasite Trypanosoma cruzi. J Struct Biol. 2005; 150: 11-22

Piñeyro MD, Parodi-Talice A, Arcari T, Robello C. Peroxiredoxins from Trypanosoma cruzi: virulence factors and drug targets for treatment of Chagas disease? Gene. 2008; 408: 45-50.
Schröder E, Littlechild JA, Lebedev AA, Errington $\mathrm{N}$, Vagin AA, Isupov MN. Crystal structure of decameric 2-Cys peroxiredoxin from human erythrocytes at $1.7 \AA$ resolution. Structure. 2000; 8: 605-615.

Schlecker T, Schmidt A, Dirdjaja N, Voncken F, Clayton C, Krauth-Siegel RL. Substrate specificity, localization, and essential role of the glutathione peroxidase-type tryparedoxin peroxidases in Trypanosoma brucei. J Biol Chem. 2005; 280: 14385 14394.

Singh N, Kumar M, Singh RK. Leishmaniasis: Current status of available drugs and new potential drug targets. Asian Pac J Trop Med. 2012; 5(6): 485-497.

Thomsen R. and Christensen MH. MolDock: A New Technique for High-Accuracy Molecular Docking. $J$ Med Chem. 2006; 49(11): 3315-3321.

Tovar J, Wilkinson S, Mottram JC, Fairlamb AH. Evidence that trypanothione reductase is an essential enzyme in Leishmania by targeted replacement of the TRYA gene locus. Mol Microbiol. 1998; 29: 653-660.

Werner T, Morris MB, Dastmalchi S, Church WB. Structural modelling and dynamics of proteins for insights into drug interactions. Adven Drug Del Rev. 2012; 64: 323-343.

Wiederstein M, Sippl MJ. ProSA-web: interactive web service for the recognition of errors in threedimensional structures of proteins. Nucleic Acid Res. 2007; 35: 407-10.

Wilkinson SR, Temperton NJ, Mondragon A, Kelly JM. Distinct mitochondrial and cytosolic enzymes mediate trypanothione-dependent peroxide metabolism in Trypanosoma cruzi. J Bio Chem. 2000; 275: 8220-8225.

Wilkinson SR, Horn D, Prathalingam SR, Kelly JM. RNA interference identifies two hydroperoxide metabolizing enzymes that are essential to the bloodstream form of the african Trypanosome. J Biol Chem. 2003; 278: 31640-31646.

World heath organization. Control of the leishmaniasis: report of a meeting of the WHO Expert Committee on the Control of Leishmaniases, Geneva; 22-26 March 2010, Report no: 949.

Wyllie S, Mandal G, Singh N, Sundar S, Fairlamb AH, Chatterjee M. Elevated levels of tryparedoxin peroxidase in antimony unresponsive Leishmania donovani field isolates. Mol Biochem Parasitol. 2010; 173: 162-164. 